\title{
APERIODIC TILINGS, POSITIVE SCALAR CURVATURE, AND AMENABILITY OF SPACES
}

\author{
JONATHAN BLOCK AND SHMUEL WEINBERGER \\ Dedicated to the memory of Ellen Block (1965 - 1991)
}

\section{INTRODUCTION}

The object of this paper is to begin a geometric study of noncompact spaces whose local structure has bounded complexity. Manifolds of this sort arise as leaves of foliations of compact manifolds and as their universal covers. We shall introduce a coarse homology theory using chains of bounded complexity and study some of its first properties.

The most interesting result characterizes when $H_{0}^{u f}(X)$ vanishes as an analogue and strengthening of Følner's amenability criterion for groups in terms of isoperimetric inequalities. (See [4].) One can view this result as producing a successful infinite Ponzi scheme on any nonamenable space. Each point, with only finite resources, gives to some of its neighbors some of these resources, yet receives more from the remaining neighbors. As one can imagine this is useful for eliminating obstructions on noncompact spaces.

This has a number of applications. We present two of them. The first produces tilings that are "unbalanced" on any nonamenable polyhedron. Unbalanced tilings are automatically aperiodic and this gives many examples of sets of tiles that tile only aperiodically. Unfortunately, imbalance is a particularly unsubtle reason for aperiodicity so that the aperiodic tilings of Euclidean space (Penrose tilings) are necessarily not accessible to our method. On the other hand, most other simply connected noncompact symmetric spaces even have unbalanced tilings using our criterion.

The second application regards characteristic numbers of manifolds whose universal covers have positive scalar curvature. We prove a converse to a theorem of Roe. We show that for any nonamenable group $\Gamma$ one can find a spin manifold with fundamental group $\Gamma$, with nonzero $\hat{A}$-genus whose universal cover has a uniformly positive scalar curvature metric of bounded geometry in the natural strict quasi-isometry class.

Received by the editors August 29, 1991.

1991 Mathematics Subject Classification. Primary 53C20, 05B45, 58G10.

The first author was supported by an NSF postdoctoral fellowship. The second author was partially supported by an NSF grant and a PYI award. 
Future papers will develop a connection to indices of elliptic operators on noncompact manifolds and with information measured by higher homology groups. These lead to new bounded geometry homotopy invariants and more quantitative information about the shape of negative scalar curvature sets. One can also apply these ideas to understanding the growth of distortion of diffeomorphisms between noncompact manifolds. (See Oliver Attie's forthcoming Courant Ph.D. thesis and also [1].) But all of these developments will be presented elsewhere.

\section{UNIFORMLY FINITE HOMOLOGY}

In this section we introduce the uniformly finite homology groups $H_{i}^{u f}(X)$ of a metric space $X$. We will derive some of its simplest properties, in particular its invariance under coarse quasi-isometry.

Let $X$ be a metric space and write $X^{i+1}$ for the $i+1$-fold cartesian product with the metric

$$
d\left(\left(x_{0}, \cdots, x_{i}\right),\left(y_{0}, \cdots, y_{i}\right)\right)=\max _{0 \leq j \leq i} d\left(x_{j}, y_{j}\right) .
$$

Also, write $\Delta$ for the multidiagonal in $X^{i+1}$. No confusion should arise from this. Let $C_{i}^{u f}(X ; \mathbf{R})=C_{i}^{u f}(X)$ denote the vector space of infinite formal sums

$$
c=\sum a_{\bar{x}} \bar{x}
$$

$\bar{x} \in X^{i+1}, a_{\bar{x}} \in \mathbf{R}$ satisfying

(1) There exists $K>0$ (depending on $c$ ) such that $\left|a_{\bar{x}}\right| \leq K$.

(2) Given $r>0$ there exists $K_{r}$ so that for all $\bar{y} \in X^{i+1}$

$$
\#\left\{\bar{x} \in B(\bar{y}, r) \mid a_{\bar{x}} \neq 0\right\} \leq K_{r} .
$$

(3) There exists $R>0$ (depending on $c$ ) so that $a_{\bar{x}}=0$ if $d(\bar{x}, \Delta)>R$.

We will sometimes regard the coefficients as describing a function from the simplices to $\mathbf{R}$ and therefore we will use notation appropriate to functions. In particular, one chain is $\leq$ another if all the coefficients are $\leq$. In a similar manner we can define $C_{i}^{\bar{u} f}(X ; \mathbf{Z})$. From now on we will let $C_{i}^{u f}(X)$ denote either $C_{i}^{u f}(X ; \mathbf{R})$ or $C_{i}^{u f}(X ; \mathbf{Z})$ unless the considerations only apply to one of the groups.

Define $\partial: C_{i}^{u f}(X) \rightarrow C_{i-1}^{u f}(X)$ by

$$
\partial\left(x_{0}, \cdots, x_{i}\right)=\sum_{j=0}^{i}(-1)^{j}\left(x_{0}, \cdots, \hat{x}_{j}, \cdots, x_{i}\right)
$$

and extend by linearity. It is easy to check that $\left(C_{\bullet}^{u f}(X), \partial\right)$ is a chain complex and we denote its homology by $H_{i}^{u f}(X)$ (or if necessary, $H_{i}^{u f}(X ; \mathbf{Z}$ ) etc. if we need to specify coefficients), and call it the uniformly finite homology of $X$. 
Note that we do not take the closure of the boundaries. In fact, the boundaries will in general not be closed in the $L^{\infty}$-norm.

A map $f: X \rightarrow Y$ between two metric spaces is said to be effectively proper Lipschitz (or EPL) if it satisfies the conditions

(1) Given $r>0$ there exists an $s>0$ so that $d(f(x), f(y))<s$ if $d(x, y)<r$. (Roe calls this uniformly bornologous. We use the term Lipschitz even though it is only Lipschitz in some coarse sense. )

(2) Given $r>0$ there exists $s>0$ so that if $d(f(x), f(y))<r$ then $d(x, y)<s$. (This condition alone is called effectively proper.)

Note that $f$ is not necessarily continuous. Also note that a coarse quasiisometry in the sense of Gromov is an EPL map.

Let $f: X \rightarrow Y$ be an EPL map. Define $f_{*}: C_{i}^{u f}(X) \rightarrow C_{i}^{u f}(Y)$ by

$$
f_{*}\left(\sum a_{\bar{x}} \bar{x}\right)=\sum a_{\bar{x}} f(\bar{x}) \text {. }
$$

Let $f_{*}$ also denote the induced map on homology. Now consider two EPL maps $f_{0}, f_{1}: X \rightarrow Y$. We say $f_{0}$ is uniformly close to $f_{1}$ if there exists $R>0$ so that $d\left(f_{0}(x), f_{1}(x)\right)<R$, for all $x \in X$.

Proposition 2.1. If $f_{0}, f_{1}: X \rightarrow Y$ are two uniformly close EPL maps, then $f_{0 *}, f_{1 *}:\left(C_{i}^{u f}(X), \partial\right) \rightarrow\left(C_{i}^{u f}(Y), \partial\right)$ are chain homotopic. In particular, $f_{0 *}=f_{1 *}$ on homology.

Proof. $f_{0}$ and $f_{1}$ being uniformly close means that $\left\{f_{0}, f_{1}\right\}: X \times\{0,1\} \rightarrow Y$ is an EPL map and as usual the proposition is reduced to showing that the two maps $i_{0}, i_{1}: X \rightarrow X \times\{0,1\}$ are chain homotopic. For this we merely write down the chain homotopy. Let $h: C_{i}^{u f}(X) \rightarrow C_{i+1}^{u f}(X \times\{0,1\})$ be defined by

$$
h\left(x_{0}, \ldots, x_{i}\right)=\sum_{j=0}^{i}(-1)^{j}\left(\left(x_{0}, 0\right), \ldots,\left(x_{j}, 0\right),\left(x_{j}, 1\right), \ldots,\left(x_{i}, 1\right)\right)
$$

and extend by linearity. Then one computes that $\partial h+h \partial=i_{1 *}-i_{0 *}$.

Two spaces $X$ and $Y$ are uniformly close if there exists $f: X \rightarrow Y$ and $g: Y \rightarrow X$, both EPL, such that $f \circ g$ and $g \circ f$ are both uniformly close to the identity.

Corollary 2.2. If $X$ and $Y$ are uniformly close then $H_{i}^{u f}(X) \cong H_{i}^{u f}(Y)$. In particular, this is the case when $X$ and $Y$ are coarsely quasi-isometric (in the sense of Gromov).

A subset $\Gamma \subset X$ is called $c$-dense or coarsely dense if there exists some number $c>0$ so that $N_{c}(\Gamma)=X$. Here $N_{c}(\Gamma)=\{y \in X \mid d(y, \Gamma)<c\}$. $\Gamma \subset X$ is called a quasi-lattice if $\Gamma$ is c-dense in $X$ and for all $r>0$ there exists $K_{r}>0$ so that

$$
\#\left\{x \in \Gamma \cap B_{r}(y)\right\} \leq K_{r}
$$


for all $y \in X$. Thus $\Gamma$ defines a class $[\Gamma] \in H_{0}^{u f}(X)$. By the corollary, the inclusion $\Gamma \subset X$ induces an isomorphism $H_{i}^{u f}(\Gamma) \cong H_{i}^{u f}(X)$. Not every metric space has a quasi-lattice, but spaces which are of bounded complexity in any reasonable sense do. See the beginning of the next section.

Let us point out that when $H_{0}^{u f}(X) \neq 0$, it tends to be rather large. For example, let $X=\mathbf{Z}$ (or $\mathbf{R}$ as they are coarse quasi-isometric). Then it is easy to check that

$$
H_{0}^{u f}(\mathbf{Z} ; \mathbf{Z}) \cong \frac{\left\{\phi: \mathbf{Z} \rightarrow \mathbf{Z} \mid\|\delta \phi\|_{\infty}<\infty\right\}}{\left\{\phi: \mathbf{Z} \rightarrow \mathbf{Z} \mid\|\phi\|_{\infty}<\infty\right\}}
$$

Here $\delta \phi(n)=\phi(n)-\phi(n-1)$ and $\|\cdot\|_{\infty}$ is the $L^{\infty}$ norm. Note, in particular, that $H_{0}^{u f}(\mathbf{Z} ; \mathbf{Z})$ is an $\mathbf{R}$ vector space via $(\lambda \cdot \phi)(n)=[\lambda \phi(n)]$. In fact, it can be shown in many circumstances that the map $H_{0}^{u f}(X ; \mathbf{Z}) \rightarrow H_{0}^{u f}(X ; \mathbf{R})$ is an isomorphism.

Finally, we have the following very useful proposition characterizing vanishing of $H_{0}^{u f}$ in terms of particular "fundamental classes."

Proposition 2.3. Suppose $\Gamma \subset X$ is a quasi-lattice. Then the following are equivalent:

(1) $H_{0}^{u f}(X ; \mathbf{Z})=0$.

(2) $H_{0}^{u f}(X ; \mathbf{R})=0$.

(3) There exists $c=\sum_{x \in \Gamma} a_{x} x$, with integer $a_{x}>0$, such that $[c]=0$ in $H_{0}^{u f}(X ; \mathbf{Z})$.

(4) There exists $c=\sum_{x \in \Gamma} a_{x} x$, with real $a_{x} \geq \varepsilon>0$, such that $[c]=0$ in $H_{0}^{u f}(X ; \mathbf{R})$.

Proof. As usual $H_{0}^{u f}(\Gamma)=H_{0}^{u f}(X)$ so we work just with $\Gamma$. We prove (3) imples (1) and (2), and (4) implies (3).

Lemma 2.4. Suppose $c=\sum_{x \in \Gamma} a_{x} x$ such that $a_{x} \geq 0$ are integers and so that $[c]=0$ in $H_{0}^{u f}(\Gamma ; \mathbf{Z})$. Then for every $x$ such that $a_{x}>0$ there exists $t_{x} \in$ $C_{1}^{u f}(\Gamma ; \mathbf{Z})$ such that $\partial\left(t_{x}\right)=x$ and the sum $\sum_{x} t_{x}$ is a locally finite bounded sum, that is, $\sum_{x} t_{x} \in C_{1}^{u f}(\Gamma ; \mathbf{Z})$.

We think of the $t_{x}$ 's as tails off to infinity and the condition that their sum is in $C_{1}^{u f}(\Gamma ; \mathbf{Z})$ is the statement that in any ball of fixed radius, the number of tails passing through is uniformly bounded.

Proof of the lemma. Suppose given such a $c$. Then $c=\partial \psi, \psi \in C_{1}^{u f}(\Gamma ; \mathbf{Z})$. $\psi$ looks like $\sum_{(x, y)} b_{(x, y)}(x, y)$. By replacing $(x, y)$ by $(y, x)$ we can assume $b_{(x, y)} \geq 0$. We induct on the maximum of the $a_{x}$ 's. Let $x_{0} \in \Gamma$ be such that $a_{x_{0}}$ realizes this maximum. There exists $x_{1} \in \Gamma$ so that the coefficient in front of $\left(x_{1}, x_{0}\right)$ in $\psi$ is positive. Then there is also an $x_{2}$ so that the coefficient of $\left(x_{2}, x_{1}\right)$ in $\psi$ is positive (since the coeffiecient in front of $x_{1}$ in $\partial \psi$ is positive). Continuing in this manner we construct $t_{x_{0}}=\left(x_{1}, x_{0}\right)+$ $\left(x_{2}, x_{1}\right)+\cdots$. Now $\partial\left(\psi-t_{x_{0}}\right)$ again satisfies the hypotheses of the theorem 
so we may iterate this procedure to construct $t_{x}$ for every $x$ with $a_{x}>0$. $\sum_{x} t_{x} \in C_{1}^{u f}(\Gamma ; \mathbf{Z})$ since the simplices making up this sum are a subset of those making up $\psi$. This proves the lemma.

To continue with the proof of the proposition, let $c$ be as in (3). Then by Lemma 2.4 , let $t_{x} \in C_{1}^{u f}(\Gamma ; Z)$ satisfy $\partial t_{x}=x$. Then any $b=\sum_{x} b_{x} x \in$ $C_{0}^{u f}(\Gamma ; \mathbf{Z})$ is the boundary $\partial\left(\sum_{x} b_{x} t_{x}\right)$. Hence $H_{0}^{u f}(\Gamma ; \mathbf{Z})=0$. The same argument shows that (3) implies (2).

To show that (4) implies (3) let $c=\sum a_{x} \cdot x \in C_{0}^{u f}(\Gamma ; \mathbf{R})$ with $a_{x} \geq \varepsilon>0$ and such that $c=\partial \psi, \psi \in C_{1}^{u f}(\Gamma ; \mathbf{R})$. Suppose supp $\psi \subset N_{r}(\Delta)$ and let $K>0$ be such that $\#\left\{B_{r}(y)\right\} \leq K$ for all $y \in \Gamma$. Set $\alpha=\frac{(K+1)}{\varepsilon}$. Define $\psi^{\prime}(x, y)=[\alpha \psi(x, y)]$. Then $\psi^{\prime} \in C_{1}^{u f}(\Gamma ; \mathbf{Z})$ and $\partial \psi^{\prime} \in C_{0}^{u f}(\Gamma ; \mathbf{Z})$. Now $\partial \psi^{\prime}(x) \geq 1$. Hence $\partial \psi^{\prime} \in C_{0}^{u f}(\Gamma ; \mathbf{Z})$ satisfies $(3)$.

\section{AMENABILITY}

Let $X$ be a metric space. It is not true that $X$ necessarily has a quasilattice. However, if $X$ has bounded geometry in some sense then it does. For example, if $X$ is a complete Riemannian manifold which has the property that its injectivity radius is bounded away from zero and its Ricci curvature is bounded from below by a constant $-(n-1) R, R>0$, then $X$ has a quasilattice. In particular any maximal subset of $X$ so that any two points are distance greater than a half (Zorn's lemma) is a quasi-lattice. Similarly, let $X$ be a polyhedron with a given triangulation $K$ and consider the standard metric defined using barycentric coordinates. Call this metric $d_{K}$. The topology induced by this metric is the same as the original topology on $X$ as long as $K$ is locally finite. Then if $K$ has bounded complexity in the sense that in any ball of radius $r>0$ there is a uniformly bounded number of simplices, then the set of vertices is a quasi-lattice. Note that if $K$ has bounded complexity, then so does $K^{\prime}$, its barycentric subdivision.

A metric space will be said to be of coarse bounded geometry if it has a quasi-lattice. For a subset $U \subset X$ let $\partial_{r} U=\{x \in X \mid d(x, U)<r$ and $d(x, X-U)<r\}$. $X$ will be called amenable if it has a quasi-lattice $\Gamma$ and for any $r, \delta>0$ there exists finite $U \subset \Gamma$ so that

$$
\frac{\#\left\{\partial_{r} U\right\}}{\#\{U\}}<\delta .
$$

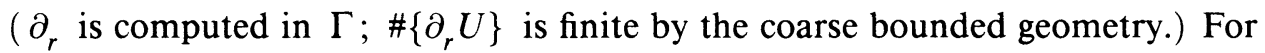
a finitely generated group $\Gamma$ with its word metric this is equivalent by Følner's condition to amenability of the group [4]. Also, if $M$ is a manifold of bounded geometry, this is equivalent to not having a linear isoperimetric inequality.

The main result of this section is the following 
Theorem 3.1. Let $X$ be a metric space of coarse bounded geometry. Then $H_{0}^{u f}(X)=0$ if and only if $X$ is not amenable. (By Proposition 2.3 it does not matter what the coefficients are.)

Proof. Let $\Gamma \subset X$ be a quasi-lattice. Then $H_{0}^{u f}(\Gamma) \cong H_{0}^{u f}(X)$ so that we might as well work with $H_{0}^{u f}(\Gamma)$. First we will show that $H_{0}^{u f}(\Gamma)=0$ implies $\Gamma$ is not amenable. Since $H_{0}^{u f}(\Gamma)=0, \phi_{0}=\sum_{x \in \Gamma} x=\partial \psi$, for some $\psi \in C_{1}^{u f}(\Gamma)$. We will show there exists $r, \delta>0$ so that

$$
\frac{\left.\#\left\{\partial_{r} U\right)\right\}}{\#\{U\}} \geq \delta
$$

for all finite subsets $U \subset \Gamma$. Let $r$ be so that $\operatorname{supp} \psi \subset N_{r}(\Delta)$. Also, let $K>0$ be such that $\#\left\{B_{r}(y)\right\} \leq K$ for all $y \in \Gamma$. Then

$$
\begin{aligned}
\#\{U\} & =\sum_{x \in U} \phi_{0}(x)=\sum_{x \in U} \partial \psi(x) \\
& =\sum_{x \in U} \sum_{y \in \Gamma} \psi(y, x)-\psi(x, y) \\
& =\sum_{x \in U} \sum_{y \in U}(\psi(y, x)-\psi(x, y))+\sum_{x \in U} \sum_{y \in \Gamma-U}(\psi(y, x)-\psi(x, y)) .
\end{aligned}
$$

The first term vanishes by symmetry. Hence we have

$$
\leq \sum_{x \in U} \sum_{y \in \Gamma-U}|\psi(y, x)-\psi(x, y)| .
$$

In order that the summand not vanish, $d(x, y)<r$ so

$$
\begin{aligned}
& =\sum_{x \in\left(\partial_{r} U\right) \cap U} \sum_{y \in \Gamma-U}|\psi(y, x)-\psi(x, y)| \\
& \leq \sum_{x \in\left(\partial_{r} U\right) \cap U} \sum_{y \in B_{r}(x)}|\psi(y, x)-\psi(x, y)| \\
& \leq \sum_{x \in\left(\partial_{r} U\right) \cap U} 2\|\psi\|_{\infty} K \leq 2 \#\left\{\partial_{r} U\right\}\|\psi\|_{\infty} K
\end{aligned}
$$

or

$$
\frac{\#\left\{\partial_{r} U\right\}}{\#\{U\}} \geq\left(2\|\psi\|_{\infty} K\right)^{-1}
$$

To prove the other direction, assume that $H_{0}^{u f}(\Gamma ; \mathbf{R}) \neq 0$. Let $\mathscr{A}=$ $\left\{\phi \in C_{0}^{u f}(\Gamma ; \mathbf{R}) \mid \exists K>0\right.$ with $\left.\phi(x) \geq K \forall x \in \Gamma\right\}$. By Proposition 2.3 $\mathscr{A} \cap \partial C_{1}^{u f}(\Gamma ; \mathbf{R})=\emptyset$ and in fact $\mathscr{A}$ is an open convex subset of $C_{0}^{u f}(\Gamma ; \mathbf{R})$. By the Hahn-Banach theorem, there exists $m \in C_{0}^{u f}(\Gamma ; \mathbf{R})^{\prime}$ of norm one, so that $m(\phi) \geq 0$ for all $\phi \in \mathscr{A}, m\left(\partial C_{1}^{u f}(\Gamma ; \mathbf{R})\right)=0$ and $m\left(\phi_{0}\right)=1$, where $\phi_{0}=\sum_{x \in \Gamma} x$. Let $\mu_{n} \in l^{1}(\Gamma)$ be a net satisfying $\left\|\mu_{n}\right\|=1, \mu_{n} \geq 0$ and $\mu_{n} \rightarrow m$ in the weak-* topology. This is possible by the weak-* density of $l^{1}(\Gamma)$ in $C_{0}^{u f}(\Gamma ; \mathbf{R})^{\prime} \cong l^{\infty}(\Gamma)^{\prime}$. We also may assume that each $\mu_{n}$ has finite support. 
Now we must show that given $r, \varepsilon>0$ that there exists $U \subset \Gamma$ so that

$$
\frac{\#\left\{\partial_{r} U\right\}}{\#\{U\}}<\varepsilon \text {. }
$$

We first prove

Lemma 3.2. Given $\varepsilon, r>0$ there exists $\phi \in l^{1}(\Gamma)$ with finite support so that $\|\phi\|_{1}=1$ and $\phi \geq 0$ and

$$
\sum_{d(x, y)<r}|\phi(x)-\phi(y)|<\varepsilon
$$

Proof of the lemma. Let

$$
C_{(1)}^{1}(\Gamma ; \mathbf{R})=\left\{\psi: \Gamma \times \Gamma \rightarrow \mathbf{R}\left|\forall r>0, \sum_{d(x, y)<r}\right| \psi(x, y) \mid<\infty\right\} .
$$

This is a topological vector space with respect to the semi-norms $\|\psi\|_{1, r}=$ $\sum_{d(x, y)<r}|\psi(x, y)|$. The topological dual of $C_{(1)}^{1}(\Gamma ; \mathbf{R})$ is easily seen to be $C_{1}^{u f}(\Gamma ; \mathbf{R}) . \quad\left(\right.$ Basically, $C_{(1)}^{1}(\Gamma ; \mathbf{R}) \cong \lim _{\leftarrow} l^{1}\left(N_{r}(\Delta)\right)$ and so $C_{(1)}^{1}(\Gamma ; \mathbf{R})^{\prime} \cong$ $\lim _{\rightarrow} l^{\infty}\left(N_{r}(\Delta)\right)=C_{1}^{u f}(\Gamma ; \mathbf{R})$.) For $\mu \in l^{1}(\Gamma)$ write $\delta \mu(x, y)=\mu(x)-\mu(y)$. Then $\delta \mu \in C_{(1)}^{1}(\Gamma ; \mathbf{R})$, and for $\mu \in l^{1}(\Gamma)$ and $\psi \in C_{1}^{u f}(\Gamma ; \mathbf{R})$ we have

$$
\langle\delta \mu, \psi\rangle=\langle\mu, \partial \psi\rangle
$$

where $\langle$,$\rangle denotes the obvious pairings.$

Now for all $\psi \in C_{1}^{u f}(\Gamma ; \mathbf{R})$ we have $\left\langle\delta \mu_{n}, \psi\right\rangle=\left\langle\mu_{n}, \partial \psi\right\rangle \rightarrow\langle m, \partial \psi\rangle=0$ since $\mu_{n} \rightarrow m$ weak-*. Hence $\delta \mu_{n} \rightarrow 0$ in the weak topology of $C_{(1)}^{1}(\Gamma ; \mathbf{R})$. Since for convex sets the weak and the strong topology closures are the same, we can take finite convex combinations of the $\mu_{n}$, say, $\phi_{n}$ so that $\delta \phi_{n} \rightarrow 0$ strongly while we still have $\left\|\phi_{n}\right\|_{1}=1$ and $\phi_{n} \geq 0$ (because $\mu_{n} \geq 0$ ). But this is the statement of the lemma. This proves the lemma.

To continue with the proof of the theorem, given $r, \varepsilon>0$ let $\phi$ be as in the lemma. Since $\phi$ has finite support, we can write

$$
\phi=\sum_{i=1}^{N} a_{i} \frac{\chi_{U_{i}}}{\#\left\{U_{i}\right\}}
$$

so that $U_{i+1} \subset U_{i}, \sum a_{i}=1$ and $a_{i} \geq 0$. Then

$$
\begin{aligned}
\varepsilon>\sum_{d(x, y)<r}|\phi(x)-\phi(y)| & =\sum_{d(x, y)<r} \sum_{i=1}^{N} a_{i} \frac{\left|\chi_{U_{i}}(x)-\chi_{U_{i}}(y)\right|}{\#\left\{U_{i}\right\}} \\
& =\sum_{i=1}^{N} \frac{a_{i}}{\#\left\{U_{i}\right\}} \sum_{d(x, y)<r}\left|\chi_{U_{i}}(x)-\chi_{U_{i}}(y)\right|
\end{aligned}
$$


but $\sum_{d(x, y)<r}\left|\chi_{U_{i}}(x)-\chi_{U_{i}}(y)\right| \geq \#\left\{\partial_{r} U_{i}\right\}$. Hence (1)

$$
\geq \sum_{i=1}^{N} a_{i} \frac{\#\left\{\partial_{r} U_{i}\right\}}{\#\left\{U_{i}\right\}}
$$

Therefore for at least one of the $i$ 's we have $\frac{\#\left\{\partial_{r} U_{j}\right\}}{\#\left\{U_{i}\right\}}<\varepsilon$ which proves the theorem.

Remark. It is interesting to note that for an $n$-manifold of bounded geometry $M, H_{0}^{u f}(M ; \mathbf{R}) \cong \Omega_{\text {bounded }}^{n}(M) / d$ (bounded), in the notation of Gromov. Hence, the theorem above implies a theorem of Gromov that $M$ is open at infinity if and only if every bounded $n$-form is $d$ (bounded). (See $[2, \S 4.1]$.)

\section{APERIODIC TILINGS}

In this section we will describe certain types of tilings of a space $X$ which are necessarily aperiodic. We then give a necessary and sufficient condition for a space to have such a tiling. Let $X$ be a polyhedron. We will say $X$ is a singular $n$ manifold if it has a triangulation $K$ such that every simplex $\sigma \in K$ is contained in an $n$-simplex. Recall the metric $d_{K}$ described at the beginning of $\S 3$. Note that $d_{K}$ is quasi-isometric to $d_{K^{\prime}}$ where $K^{\prime}$ is the barycentric subdivision of $K$.

Let $(X, d)$ be a metric space which is a singular $n$-manifold and let $K$ be a triangulation so that $d$ is quasi-isometric to $d_{K}$. A set of tiles $(\mathscr{T}, \mathscr{F})$ is a finite collection of compact metric singular $n$ manifolds $t \in \mathscr{T}$ and a collection of sub singular $(n-1)$ manifolds $f \in \mathscr{F}$, together with an opposition function $o: \mathscr{F} \rightarrow \mathscr{F}$. A metric singular $n$ manifold $X$ is tiled by the set $(\mathscr{T}, \mathscr{F})$ if there is a decomposition of $X$ as $\cup_{\lambda} t_{\lambda}$ where each $t_{\lambda}$ is isometric to one of the tiles in $\mathscr{T}$ and such that if two tiles $t_{\lambda}, t_{\mu}$ intersect, then they intersect in $f \in t_{\lambda}$ and $o(f) \in t_{\mu}$ and such that there are no free faces.

A finite set of tiles $(\mathscr{T}, \mathscr{F})$ is semibalanced if there exists a weight function $w: \mathscr{F} \rightarrow \mathbf{Z}$ such that

(1) $w(o(f))=-w(f)$.

(2) For all $t \in \mathscr{T}, \sum_{f \in t} w(f) \geq 0$.

The set is called unbalanced if the sum in (2) is $>0$. The impurity set $D$ of a semibalanced tiling is the collection of centers of gravity of the set of positive weight tiles. (The center of gravity of a tile is any point in the tile.) This defines a well-defined element in $H_{0}^{u f}(X ; \mathbf{Z})$.

The following proposition states that a semibalanced set of tiles can only tile aperiodically.

Proposition 4.1. If $X$ has a semibalanced tiling by $(\mathscr{T}, \mathscr{F})$ with impurity $D \neq$ $\emptyset$, then there exists no cocompact tile preserving action on $X$. 
Proof. Suppose $\Gamma$ is a group acting on $X$ preserving tiles and such that the quotient is compact. Then the finite sum

$$
\sum_{t_{\lambda} \subset X / \Gamma} \sum_{f \in t_{\lambda}} w(f)>0
$$

If, instead, we sum over the faces, then

$$
\sum_{t_{\lambda} \subset X / \Gamma} \sum_{f \in t_{\lambda}} w(f)=\sum_{f \in X / \Gamma} w(f)+w(o(f))=0
$$

Theorem 4.2. Let $X$ be a noncompact singular $n$-manifold $n \geq 2$ with triangulation $K$, and metric $d_{K}$ and assume $X$ is of finite complexity. Then

(1) $X$ has an unbalanced tiling if and only if $X$ is nonamenable.

(2) Let $D \subset X$ be such that there exists an $r>0$ so that for any two elements $x$ and $y$ in $D, d(x, y)>r$. Then $X$ has a semibalanced tiling with impurity $D$ if and only if $[D]=0$ in $H_{0}^{u f}(X ; \mathbf{Z})$.

Proof. First we prove (2). Suppose $X$ is tiled by $(\mathscr{T}, \mathscr{F})$ an unbalanced set of tiles with weight function $w$. Let $D$ be the impurity set of the tiling, so $D$ defines an element in $H_{0}^{u f}(X ; \mathbf{Z})$. For each pair of elements $x$ and $y$ in $D$, let $t_{x}$ and $t_{y}$ be the tiles containing $x$ and $y$ and define $a_{(x, y)}=w(f)$ where $f$ is the face of $t_{y}$ in the intersection $t_{x}$ and $t_{y}$, or equal to 0 if they have no common face. Then

$$
\partial \sum_{(x, y)} a_{(x, y)}(x, y)=\sum_{x \in D}\left(\sum_{f \in t_{x}} w(f)\right)>0 .
$$

Hence $[D]=0$ in $H^{u f}(D ; \mathbf{Z})=0$ by Proposition 2.3 and so is also zero in $H_{0}^{u f}(X ; \mathbf{Z})$. The same argument implies the "only if" part of $(1)$.

Now let $D$ be as in (2). By subdividing $K$ enough times we may assume that each $n$ simplex in $X$ contains at most one element of $D$. We may even assume that each element of $D$ is a vertex of $K$. Let $V$ be the set of vertices of $K$. By assumption, $D$ is a boundary so let $D=\partial \psi$ where $\psi \in C_{1}^{u f}(V ; \mathbf{Z})$. By replacing the chain $(x, y) \in C_{1}^{u f}(V ; \mathbf{Z})$ by $\left(x_{0}, x_{1}\right)+\left(x_{1}, x_{2}\right)+\cdots+$ $\left(x_{m-1}, x_{m}\right)$ where $x_{0}=x, x_{m}=y, d\left(x_{j}, x_{j+1}\right)=1$, we may assume that supp $\psi \subset N_{1}(\Delta)$.

Now let $K^{\prime}$ be the barycentric subdivision of $K$. For $x \in V$ let $* x=$ $\cup_{\sigma^{n} \in K^{\prime}, x \in \sigma^{n}} \sigma^{n}$. Since $K^{\prime}$ has finite complexity, there are only a finite number of combinatorial types of $* x$ as $x$ runs over $V$. Now for each edge $e=$ $(x, y) \in K$, let $* e=* x \cap * y$. As with the $* x$ 's, there are also a finite number of combinatorial types of $* e$ 's. Let $\mathscr{T}^{\prime}$ be the collection of combinatorial types of $* x$ 's and $\mathscr{F}^{\prime}$ the types of $* e$ 's. Certainly, $X$ is tiled by the set $\left(\mathscr{T}^{\prime}, \mathscr{F}^{\prime}\right)$. We now derive from $(\mathscr{T}, \mathscr{F})$ and $\psi$ a new set of tiles $(\mathscr{T}, \mathscr{F})$ and a weight function making $(\mathscr{T}, \mathscr{F})$ semi-balanced with impurity $D$. First we assign weights to the faces of each tile in this tiling of $X$ as follows. For a 
tile $* x$, with faces $* e_{x}$ where $e_{x}$ is an edge containing $x$, assign the weight $w\left(* e_{x}\right)= \pm \psi\left(e_{x}\right)$ where the \pm is chosen according to whether $x$ is the final or initial point of $e_{x}$. Then

$$
\sum_{f \in * x} w(f)=\partial \psi(x)=\left\{\begin{array}{cc}
1 & x \in D \\
0 & \text { if not }
\end{array}\right\} .
$$

Now construct the new set of tiles as follows. Consider the set of tiles $t \in X$ together with their configuration of faces and weight functions. This is a finite set since the initial set of tiles were finite and since $\psi \in C_{1}^{u f}(V ; \mathbf{Z})$, that is $\psi$ assumes only a finite number of different values. This will basically be our new set of tiles. The only thing left to do is to make the faces with different weights combinatorially distinct which we can do since $n-1 \geq 1$. Thus we arrive at a new set of tiles $(\mathscr{T}, \mathscr{F})$ and the tiling is seen to have impurity $D$.

The proof of the "if" part of (1) is the same.

This construction may also be carried out in some situations on Riemannian manifolds. For example,

Proposition 4.4. Let $\widetilde{M} \rightarrow M$ be a covering space of a compact Riemannian manifold $M$, and consider $\widetilde{M}$ to have the lifted metric. Then $\widetilde{M}$ has an unbalanced tiling if and only if the covering group is not amenable.

There is also a statement about semibalanced tilings. The proof of this is similar to that of the previous proposition except that the combinatorics is handled via the group and a fundamental domain.

In the case where the group of the cover contains a nonabelian free group one can actually produce unbalanced tilings effectively. We do not know how to do this in general. (We remind the reader that Grigorchuk has produced examples of nonamenable groups without free subgroups [4].)

\section{ApPlications to POSITIVE SCALAR CURVATURE}

Let $\pi: \widetilde{M} \rightarrow M$ be a covering space over a compact manifold $M$ with an amenable covering group $\Gamma$. Then if $\widetilde{M}$ is spin the $\hat{A}(M)$ is an obstruction to finding a metric of positive scalar curvature on $\widetilde{M}$ in the same strict quasi-isometry class as the pull back metric from $M$ according to Roe [5, II, Proposition 3.3]. The following proposition shows that the necessity of the amenability hypothesis is not at all a weakness of Roe's methods, but is inherent to the problem.

Proposition 5.1. Let $\Gamma$ be a nonamenable group. Then there exists a $\Gamma$-covering space $\pi: \widetilde{M} \rightarrow M$ so that $M$ is compact spin manifold, $\hat{A}(M) \neq 0$ and so that $\widetilde{M}$ has a metric of positive scalar curvature in the same strict quasi-isometry class as the pullback metric from $M$.

In fact we will prove something slightly more general.

Lemma 5.2. Let $W$ be a $4 k$-dimensional Riemannian manifold of bounded geometry $(k>1)$ which has a metric of positive scalar curvature. Let $L$ denote a 
compact simply connected spin manifold of dimension $4 k$ with $\hat{A}(L) \neq 0$ and diameter $d$. Let $D \subset W$ be a subset such that any two points are separated by at least $2 d$. D then defines a class $[D] \in H_{0}^{u f}(W ; \mathbf{Z})$ (since $W$ has bounded geometry). Then if $[D]=0$ in $H_{0}^{u f}(W ; \mathbf{Z})$ the manifold $W_{D}(D L)$ has a metric of positive scalar curvature in the same strict quasi-isometry class as $W_{D}(D L)$.

Note that the strict quasi-isometry class of $W \#_{D} D L$ is independant of the metric on $L$. As an example, $L$ could be $K \times K$ where $K$ is a Kummer surface. The proposition follows from the lemma by letting $M$ be a spin 8manifold with positive scalar curvature and fundamental group $\Gamma$. Such an $M$ can be produced by taking the product of an arbitrary spin 5-manifold with the correct fundamental group and a round sphere of tiny radius. Then $\hat{A}(M \# L) \neq 0, M \# L$ is spin, has fundamental group $\Gamma$, and its universal cover has metric of positive scalar curvature in the strict quasi-isometry class as the pullback metric.

To prove the lemma we will apply the theorem of Gromov and Lawson that a manifold obtained from a manifold of positive scalar curvature from performing surgeries in codimension $\geq 3$ also carries a metric of positive scalar curvature, [3, Theorem A]. It also follows from the proof of their theorem that we can leave the metric on the original manifold unchanged in the complement of the region where the surgeries are carried out. Now let $D$ be as above and assume $[D]=0$ and let $D=\partial \psi=\partial \sum_{(x, y)} b_{(x, y)}(x, y)$. Let $r$ be such that supp $\psi \subset N_{r}(\Delta)$. For a single term $b_{(x, y)}(x, y)$ we can construct a cobordism from $W$ to $W \#_{(y-x)} b_{(x, y)} L$ by starting with $(W \times I)$ and connect summing $W \times\{1\} \#_{y}\left(b_{(x, y)} L \times\{0\}\right) \#_{x}\left(-b_{(x, y)} L \times\{1\}\right)$. Now it is easy to see that the spin structures on $W$ and $L$ extend over this cobordism. Hence, by applying the argument in the proof of [3, Theorem B], we see that $W_{(y-x)} b_{(x, y)} L$ can be obtained from $W$ by applying surgeries in codimension $\geq 3$. Thus $W \#_{(y-x)} b_{(x, y)} L$ has a metric of positive scalar curvature and so that the metric has not changed at distances greater than say $2 r$ from $x$ and $y$. Continuing in this manner for each term $b_{(x, y)}(x, y)$ we construct a metric of positive scalar curvature on $W \#_{D} D L$. (A crucial point is that the theorem of Smale on the structure of cobordisms, cited in the proof of [3, Theorem B], also works in the relative case.) So that once we have done our work in one region of the the manifold $W$, of which there is only a finite amount since $\psi \in C_{1}^{u f}(W ; \mathbf{Z})$, we can leave this region forever and never disturb it again. The metric we have constructed is in the same strict quasi-isometry class as the original metric since all the surgeries take place on a uniformly large scale and since $\psi \in C_{1}^{u f}(W ; \mathbf{Z})$.

\section{ACKNOWLEDGMENT}

The second author would like to thank Jeff Cheeger for a conversation several years ago regarding the contents of [5]. Both authors would like to thank the Courant Institute for its hospitality. The authors would also like to thank 
Devorah Segal for pointing out the similarity between our work and Ponzi's original unsuccessful schemes.

\section{REFERENCES}

1. O. Attie, J. Block, and S. Weinberger, Characteristic classes and distortion of diffeomorphisms, J. Amer. Math. Soc. 5 (1992), 919-921.

2. M. Gromov, Hyperbolic manifolds, groups and actions, Riemann Surfaces and related topics: Proceedings of the 1978 Stony Brook Conference, Ann. of Math. Stud., vol. 97, Princeton University Press, Princeton, NJ, 1980.

3. M. Gromov and B. Lawson, The classification of simply connected manifolds of positive scalar curvature, Ann. of Math. (2) 111 (1980), 423-434.

4. A. Paterson, Amenability, Math. Surveys Monographs, Amer. Math. Soc., Providence, RI, 1988.

5. J. Roe, An index theorem on open manifolds. I, II, J. Differential Geom. 27, (1988), 87-136.

6. $\_$Exotic cohomology and index theory on complete Riemannian manifolds, Preprint (1990).

Mathematics Department, University of Pennsylvania, Philadelphia, Pennsylvania 19104

E-mail address: blockj@math.upenn.edu

Mathematics Department, University of Chicago, Chicago, Illinois 60607

E-mail address: shmuel@zaphod.uchicago.edu

Current address, Jonathan Block and Shmuel Weinberger: Courant Institute of the Mathematical Sciences, New York University, New York, New York 10012 